\title{
TLR4 gene expression in pig populations and its association with resistance to Escherichia coli F18
}

\author{
J. Wang ${ }^{1}$, Z.Y. Pan ${ }^{1}$, X.R. Zheng ${ }^{1}$, Z.C. $\mathrm{Wu}^{1}$, X.M. Su${ }^{1}$, G.Q. Zhu ${ }^{2}$, \\ X.G. Huang ${ }^{3}$, S.L. $\mathrm{Wu}^{1}$ and W.B. Bao ${ }^{1}$ \\ ${ }^{1}$ College of Animal Science and Technology, \\ Key Laboratory for Animal Genetics, Breeding, \\ Reproduction and Molecular Design of Jiangsu Province, \\ Yangzhou University, Yangzhou, China \\ ${ }^{2}$ College of Veterinary Medicine, Yangzhou University, Yangzhou, China \\ ${ }^{3}$ Suzhou Taihu Pig Breeding Center, Jiangsu Province, Suzhou, China \\ Corresponding authors: W.B. Bao / S.L. Wu \\ E-mail: wenbinbao74@yahoo.com / pigbreeding@163.com
}

Genet. Mol. Res. 12 (3): 2625-2632 (2013)

Received August 7, 2012

Accepted March 15, 2013

Published July 30, 2013

DOI http://dx.doi.org/10.4238/2013.July.30.1

\begin{abstract}
TLR4 is the main recognition receptor of bacterial lipopolysaccharides, which play an important role in innate and adaptive immunity. We used real-time PCR to analyze the tissue expression profile and differential expression of TLR4 in 4 pig populations (Escherichia coli F18-resistant Sutai, E. coli F18-sensitive Sutai, Large White, Meishan), in order to determine the role that the TLR4 gene plays in resistance to $E$. coli F18. We found that TLR4 expressed consistently in the 4 populations, with relatively high levels in immune tissues and the highest level in the lung. Generally, the expression of TLR4 in E. coli F18-sensitive individuals was the highest, followed by that in E. coli F18-resistant, Large White and Meishan. In the spleen, lung, kidney, lymph nodes, and thymus gland, TLR4 expression is significantly higher in the E. coli F18-sensitive than in the other 3 populations; there were no significant differences among E. coli F18-resistant Sutai, Large White, and Meishan. In addition, Gene Ontology and pathway analysis showed that TLR4 takes part in the inflammatory response. We found that porcine TLR 4 has consistent tissue specificity in each breed, and downregulation of expression of the TLR4
\end{abstract}


gene is related to resistance to $E$. coli $\mathrm{F} 18$ in weaning piglets.

Key words: Escherichia coli F18; Pig; Real-time PCR; TLR4 gene

\section{INTRODUCTION}

To date, the post-weaning diarrhea or edema disease caused by Escherichia coli F18, a type of Gram-negative bacteria, has caused great economic losses in the pig industry. Lipopolysaccharides (LPS) from E. coli F18 can be recognized by Toll-like receptor 4 (TLR4). However, the relationship between $E$. coli $\mathrm{F} 18$ disease and porcine TLR 4 expression is still unclear.

In recent years, much research has focused on the genetic variations of TLR4, part of which has reported that the gene is involved in susceptibility and resistance to diseases to a certain extent (Leveque et al., 2003). However, studies on the expression of TLR4 affected by LPS in various cells have emerged in multitude, showing that LPS can upregulate the expression of TLR4 (Yang et al., 2010; Guzzo et al., 2012), as well as showing phyletic specificity and histiocyte-specificity. However, the impacts of TLR4 are like a double-edged sword. On one hand, TLR4 signaling in macrophages activates hundreds of genes that contribute to defense against infection by bacteria, such as Mycobacterium tuberculosis (Abel et al., 2002), Pneumococcal (Klein et al., 2008), and Salmonella (Roy et al., 2006). On the other hand, by initiating the secondary inflammatory signaling pathways, TLR4 can amplify acute inflammatory responses and cause severe disease, such as atopic dermatitis (Penders et al., 2010) and acute lung injury (ALI) (Wang et al., 2011). Therefore, the role of TLR 4 is so important in disease. Although the mechanism is still unknown, the levels of $T L R 4$ expression can play a crucial role in it. Therefore, detecting TLR 4 expression can be important in further research on the mechanism of TLR4.

In general, TLR4 is widespread in T lymphocytes, B lymphocytes, leukocytes, macrophages, intestinal epithelial cells, and respiratory epithelial cells (Zhang et al., 2005) and expressed at a low level in the intestinal mucosa (Abreu et al., 2001; Otte et al., 2004). However, research on the expression of TLR4 in swine is scarce, with only some reports showing a high level in lung (Qiu et al., 2007). Considering that the resistance to pathogens varies between foreign and Chinese domestic breeds, the examination of their TLR4 expression is of great significance.

As a key linkage, TLR4 plays a very important role between natural and specific immunity. The level of TLR4 expression is closely connected with the resistance to disease. Considering the initial studies on the TLR4 gene, the detection of TLR4 gene expression in foreign, hybrid, and Chinese domestic pig breeds is an attractive route for the analysis of differences in genetic resistance and mechanisms with regard to $E$. coli F18 infection in 3 pig breeds (Large White, Sutai, and Meishan).

The pathogenesis of F18 antigen is that after E. coli strains enter the pig's intestinal tract, they strongly adhere to intestinal epithelial cells via their pili and bind to the F18 receptors in the brush border membrane of intestinal epithelial cells. They then settle, propagate, produce enterotoxins, and cause diseases in piglets (Bertin and Ducher-Suchaux, 1991; Nagy and Fekete, 1999). Using candidate gene approach and linkage analysis, Vogeli et al. (1997) demonstrated that alpha $(1,2)$-fucosyltransferase gene $(F U T 1)$ on chromosome $6 \mathrm{q} 11$ is a candidate gene controlling the adhesion to F18 receptor. The research of Meijerink et al. (1997) showed that there is a G/A mutation at position M307 of the FUT1 gene, with G being predominant over A. In other words, pigs with genotype AA are resistant to ETEC F18 and pigs with genotype GG or the heterozygote AG are sensitive to ETEC F18. Breeding for disease resistance could be implemented by marker-assisted selection using the FUT1 gene as the ETEC F18R candidate gene. Using a small number of 
FUT1 AG type (9.2\%) individuals detected from Sutai pigs (Bao et al., 2008), our group conducted proper selection and assortative mating. Small intestine epithelial cells of resistant (AA genotype) and sensitive (AG and GG genotypes) pigs were selected to test the adhesion capability of the wildtype $E$. coli expressing F18ab fimbriae, the recombinant $E$. coli expressing F18ac fimbriae, or the recombinant $E$. coli secreting and surface-displaying the FedF subunit of F18ab fimbriae, respectively (Wu et al., 2007). After 5 years of breeding, we now established resistant (AA genotype) and sensitive (AG and GG genotypes) resource populations to ETEC F18 in our Sutai pig population. Thereby, Sutai pig can be considered as the linkage between foreign and domestic breeds in the fields of investigating TLR4 gene expression and E. coli F18 infection in pigs. The expression of the TLR4 gene in different pig breeds can be also helpful in the development of research on the functions of the TLR4 gene and its potential relationship with resistance to E. coli F18.

\section{MATERIAL AND METHODS}

\section{Experimental materials and sample collection}

Large White, Sutai, and Meishan pigs (35 days old) were obtained from the Engineering Research Centre for Molecular Breeding of Pig in Changzhou City of Jiangsu Province, E. coli F18-resistant/sensitive population in Suzhou Taihu Pig Breeding Center, and Meishan Pig Conservation Breeding Company, respectively. Each population included 8 piglets. After sacrifice, heart, liver, spleen, lung, kidney, stomach, thymus, lymph node, jejunum, duodenum, and muscle were collected in 1.5-mL Eppendorf nuclease-free tubes, stored immediately in liquid nitrogen, and then placed in a low-temperature freezer $\left(-80^{\circ} \mathrm{C}\right)$ until further study.

\section{Real-time PCR primer design}

Using the Primer Express 2.0 software, TLR4 primers were designed on the basis of the sequence of AB232527 (http://www.ncbi.nlm.nih.gov/) in GenBank and synthesized by Shanghai Invitrogen Biotechnology Co., Ltd. GAPDH was used as an internal control to normalize all of the threshold cycle $(\mathrm{Ct})$ values of other tissue products. Primer sequences for amplification of TLR4 and GAPDH are listed in Table 1.

\begin{tabular}{llc}
\multicolumn{2}{l}{ Table 1. Primers used for real-time PCR. } \\
\hline Gene & Sequence & Expected length (bp) \\
\hline$T L R 4$ & Forward primer: 5'-CAGATAAGCGAGGCCGTCATT-3' & 113 \\
GAPDH & Reverse primer: 5'-TTGCAGCCCACAAAAAGCA-3' & 187 \\
& Forward primer: 5'-ACATCATCCCTGCTTCTACTGG-3' \\
& Reverse primer: 5'-CTCGGACGCCTGCTTCAC-3' & 187 \\
\hline
\end{tabular}

\section{RNA extraction and reaction system and conditions for fluorescence quantitative PCR}

Total RNA was extracted from various swine tissues (50-100 mg) using Trizol reagent (TaKaRa Biotechnology Dalian Co., Ltd.). Precipitated RNA was resuspended in $20 \mu \mathrm{L}$ RNase-free $\mathrm{H}_{2} \mathrm{O}$ and then stored at $-80^{\circ} \mathrm{C}$. RNA quality and quantity were assessed by agarose gel electrophoresis and UV spectrophotometer, respectively. 
The 10- $\mu \mathrm{L}$ reaction mixture for cDNA synthesis contained the following: $2 \mu \mathrm{L} 5 \mathrm{X}$ PrimerScript Buffer, $0.5 \mu \mathrm{L}$ PrimerScript RT Enzyme Mix I, $0.5 \mu \mathrm{L}$ Oligo dT, $0.5 \mu \mathrm{L}$ random 6-mers, $500 \mathrm{ng}$ total RNA, and RNase-free $\mathrm{H}_{2} \mathrm{O}$ to make up the final volume of $10 \mu \mathrm{L}$. The reaction was carried out at $37^{\circ} \mathrm{C}$ for $15 \mathrm{~min}$ and then at $85^{\circ} \mathrm{C}$ for $5 \mathrm{~s}$.

Real-time PCR amplification was performed in a $20-\mu \mathrm{L}$ reaction mixture containing 1 $\mu \mathrm{L}$ cDNA (100-500 ng), $0.4 \mu \mathrm{L} 10 \mu \mathrm{M}$ of each forward and reverse primer, $0.4 \mu \mathrm{L} 50 \mathrm{X}$ ROX

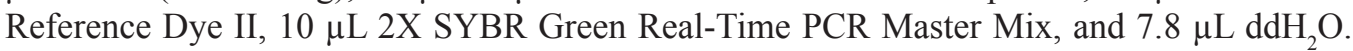
PCR conditions were $95^{\circ} \mathrm{C}$ for $15 \mathrm{~s}$, followed by 40 cycles of $95^{\circ} \mathrm{C}$ for $5 \mathrm{~s}$ and $62^{\circ} \mathrm{C}$ for $34 \mathrm{~s}$. The dissociation curve was analyzed after amplification. A peak of Tm at $85 \pm 0.8^{\circ} \mathrm{C}$ on the dissociation curve was used to determine the specificity of PCR amplification. The Tm value for each sample was the average of the real-time PCR data for triplicate samples.

\section{Data processing and analysis}

The $2^{-\Delta \Delta C t}$ method was used to process the real-time PCR results (Livak and Schmittgen, 2001). Statistical analyses were carried out using the SPSS 15.0 software (SPSS Inc, Chicago, IL, USA), and a $t$-test was carried out to determine the significance of differences in mRNA expression between different breeds.

\section{RESULTS}

\section{The purity and integrity of total RNA}

Total RNA samples were assayed using $1 \%$ agarose gel electrophoresis. Three bands, representing $28 \mathrm{~S}, 18 \mathrm{~S}$, and $5 \mathrm{~S}$, were observed with no bands from DNA contamination or significant degradation. RNA purity was also examined on a UV spectrophotometer. The $\mathrm{A}_{260}$ ' $\mathrm{A}_{280}$ ratios of the samples were 1.8-1.9.

\section{Fluorescence quantitative PCR amplification curve and melting curve}

The PCR amplification curve and the dissociation curve for the TLR4 gene showed good repetition, and a single specific peak was observed with the real-time PCR products for the TLR 4 gene with no primer dimers or nonspecific reaction products. The standard curves for the TLR4 and GAPDH genes indicated that the amplification efficiencies of the target gene and the reference gene were almost the same, so that the $2^{-\Delta \Delta \mathrm{Ct}}$ method could be applied for quantitative calculation (Figure S1). Data were analyzed by SPSS 11.5 and shown as means $\pm \mathrm{SD}$. The $t$-test was used to inspect the significance of expression in different populations.

\section{Results of $T L R 4$ gene expression in different tissues and populations}

Using the established SYBR green real-time quantitative PCR method described above, the expression levels of TLR4 were examined in various tissues in this study. The expression level of TLR4 in the heart of the resistance group was defined as 1.0. As the results shown in Table 2 and Figure S2, the TLR4 gene was expressed in all tissues tested with the highest level in lung. In general, there was high expression in immune tissues, including thy- 


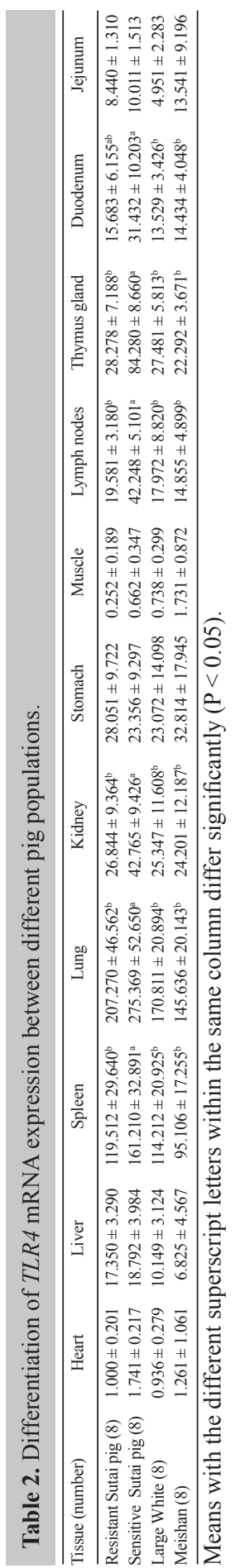


mus gland, lymph node, and spleen. In addition, the expression of the TLR4 gene was the highest in sensitive Sutai, followed by resistant Sutai, Large White, and Meishan pigs. There was a consistent expression profile in the tissues of spleen, lung, kidney, lymph nodes, and thymus gland, in which TLR4 expression in E. coli $\mathrm{F} 18$-sensitive individuals was significantly higher than that in other breeds $(\mathrm{P}<0.05)$. In duodenum, the expression of the TLR4 gene in E. coli F18-sensitive ones was significantly higher than that in Large White and Meishan $(\mathrm{P}<0.05)$. There was no significant relationship between other tissues in the 4 populations.

\section{TLR4 Gene Ontology (GO) and pathway analysis}

According to GO database classifications generated by the US National Center for Biotechnology Information (NCBI) and the KEGG database (http://www.genome.ad.jp/kegg/), the main function of the TLR4 gene has been determined. As shown in Table S1, TLR 4 takes part in 97 biological function processes, mainly integral to membranes, and is involved in the immune response as well as response to LPS. In addition, TLR4 participates in 15 pathways, such as Toll-like receptor signaling pathway, pertussis, and tuberculosis (Table S2). The results showed that it had the potential to participate in 3 pathways related to recognition of Gramnegative bacteria, where this important information could be used as a basis for further study.

\section{DISCUSSION}

Medzhitov et al. (1997) first identified and cloned the human TLR4 gene, which has a higher degree of expression in spleen, endothelial cells, macrophages, neutrophils, and dendritic cells. In swine, Bao et al. (2011) reported that TLR4 expressed in all tissues tested, with the highest expression in lung and a relatively high expression level in immune tissue. In this study, TLR 4 expressed in all tissues among 3 breeds with the highest expression level in the lungs and a relatively high level in spleen, kidney, thymus, lymph tissue, and the results were in agreement with Bao et al. (2011). The results showed that there was a consistent tissue expression pattern in Sutai, Meishan, and Large White, indicating that porcine TLR4 has consistent tissue specificity in each breed.

This study showed that in high TLR4 expressing tissues of Sutai such as lung, spleen, kidney, lymph node, and thymus, the expression of the TLR4 gene in E. coli F18-sensitive individuals was significantly higher than that in resistant individuals $(\mathrm{P}<0.05)$, indicating that $T L R 4$ has a certain impact on porcine Gram-negative bacteria such as E. coli F18. Thus, we can draw the conclusion that upregulation of TLR 4 expression can increase the risk of infection by $E$. coli $\mathrm{F} 18$. On the other hand, the results indicated that downregulation of TLR4 expression may be related to resistance to $E$. coli $\mathrm{F} 18$. On the basis of the GO and KEGG databases, the results of functional analysis of the TLR4 gene implied more detailed relationships between TLR 4 and $E$. coli $\mathrm{F} 18$, such as response to bacteria, inflammatory response, and LPS receptor activity.

Current reports show that individuals with TLR4 gene deficiency or lower expression have a lower rate of infection caused by pathogens (Oyama et al., 2004; Van Linthout et al., 2011). However, some reports show that antigen peptide from pathogens such as LPS can stimulate dendritic cells to overexpress TLR4 (An et al., 2002), which will cause continued expansion of inflammation by activating NF-KB (Bauer et al., 2007). It is worth discussing what is the kind of causal relationship between the upregulation of TLR4 gene expression and 
E. coli infection. It is still unknown whether it is overexpression of TLR4 that decreases the body's resistance to $E$. coli, or the E. coli infection that induces TLR4 gene expression, making the body more sensitive to $E$. coli. The next step is to analyze the relationship between TLR 4 gene and E. coli systematically.

Some previous studies showed that the downregulation of TLR4 expression was closely associated with human disease resistance, including cancer, pulmonary disease, and dermatitis diseases (Wieland et al., 2005; Sun et al., 2008; Penders et al., 2010), which implied that the downregulation of TLR 4 was related to general resistance. In addition, some research has also shown that Chinese indigenous pig breeds such as Meishan, one of the materials of this study, have a strong general resistance (He et al., 2001; Clapperton et al., 2005). In this study, the lower expression of TLR4 in the vast majority of Meishan pigs further implied that the downregulation of TLR 4 expression had a correlation with general resistance.

Compared to E. coli F18-sensitive Sutai groups, TLR4 mRNA was downregulated in E. coli F18-resistant ones in the lung, spleen, kidney, lymph nodes, and thymus. In addition, it is worthwhile noting that there were no differences between E. coli F18-resistance and the other 2 breeds Large White and Meishan, which showed that when screening the diseaseresistance groups, it is impossible to improve the body's inherent general resistance just by focusing on a special pathogen such as E. coli F18. TLR4, as a recognition of antigen, not only can trigger innate immunity but can also activate specific immunity, acting as a bridge in the key link connecting innate immunity with specific immunity (Beutler, 2005). These test results suggested that the TLR4 gene may be closely associated with the genetic basis of species innate immune response. Aiming at a specific pathogen for breeding by molecular methods cannot improve the species' innate immune response. There is a need for further analysis of the innate immune pathways and immune regulatory mechanism to provide guidance and basis for improving the body's immune response.

\section{ACKNOWLEDGMENTS}

Research supported by the National Natural Science Funds (\#31172183), the Prospective Joint Research Project of Jiangsu Province (\#BY2012157), the Science and Technology Supporting Project of Jiangsu Province (\#BE2012330), and the Priority Academic Program Development of Jiangsu Higher Education Institutions (PAPD).

\section{Supplementary material}

\section{REFERENCES}

Abel B, Thieblemont N, Quesniaux VJ, Brown N, et al. (2002). Toll-like receptor 4 expression is required to control chronic Mycobacterium tuberculosis infection in mice. J. Immunol. 169: 3155-3162.

Abreu MT, Vora P, Faure E, Thomas LS, et al. (2001). Decreased expression of Toll-like receptor-4 and MD-2 correlates with intestinal epithelial cell protection against dysregulated proinflammatory gene expression in response to bacterial lipopolysaccharide. J. Immunol. 167: 1609-1616.

An H, Yu Y, Zhang M, Xu H, et al. (2002). Involvement of ERK, p38 and NF-kappaB signal transduction in regulation of $T L R 2, T L R 4$ and TLR9 gene expression induced by lipopolysaccharide in mouse dendritic cells. Immunology 106: 38-45.

Bao WB, Wu SL, Musa HH, Zhu GQ, et al. (2008). Genetic variation at the alpha-1-fucosyltransferase (FUT1) gene in Asian wild boar and Chinese and Western commercial pig breeds. J. Anim. Breed. Genet. 125: 427-430.

Bao WB, Ye L, Pan ZY, Zhu J, et al. (2011). Analysis of polymorphism in the porcine TLR4 gene and its expression related 
to Escherichia coli F18 infection. Czech J. Anim. Sci. 56: 475-482.

Bauer S, Hangel D and Yu P (2007). Immunobiology of toll-like receptors in allergic disease. Immunobiology 212: 521-533. Bertin AM and Duchet-Suchaux MF (1991). Relationship between virulence and adherence of various enterotoxigenic Escherichia coli strains to isolated intestinal epithelial cells from Chinese Meishan and European large white pigs. Am. J. Vet. Res. 52: 45-49.

Beutler B (2005). The Toll-like receptors: analysis by forward genetic methods. Immunogenetics 57: 385-392.

Clapperton M, Bishop SC and Glass EJ (2005). Innate immune traits differ between Meishan and Large White pigs. Vet. Immunol. Immunopathol. 104: 131-144.

Guzzo C, Ayer A, Basta S, Banfield BW, et al. (2012). IL-27 enhances LPS-induced proinflammatory cytokine production via upregulation of TLR4 expression and signaling in human monocytes. J. Immunol. 188: 864-873.

He WM, Jiang YF, Luo WH, Huang JW, et al. (2001). Pathogenic diagnosis and epidemiological investigation on edema disease of pigs. Acta Agric. Bor.-Occid. Sin. 10: 1-5.

Klein M, Obermaier B, Angele B, Pfister HW, et al. (2008). Innate immunity to Pneumococcal infection of the central nervous system depends on toll-like receptor (TLR) 2 and TLR4. J. Infect. Dis. 198: 1028-1036.

Leveque G, Forgetta V, Morroll S, Smith AL, et al. (2003). Allelic variation in TLR4 is linked to susceptibility to Salmonella enterica serovar Typhimurium infection in chickens. Infect. Immun. 71: 1116-1124.

Livak KJ and Schmittgen TD (2001). Analysis of relative gene expression data using real-time quantitative PCR and the $2^{-\Delta \Delta C \mathrm{Ct}}$ method. Methods 25: 402-408.

Medzhitov R, Preston-Hurburt P and Janeway CA Jr (1997). A human homologue of the Drosophila Toll protein signals activation of adaptive immunity. Nature 388: 394-397.

Meijerink E, Fries R, Vogeli P, Masabanda J, et al. (1997). Two $\alpha(1,2)$ fucosyltransferase genes on porcine chromosome 6q11 are closely linked to the blood group inhibitor (S) and Escherichia coli F18 receptor (ECF18R) loci. Mamm. Genome 8: 736-741.

Nagy B and Fekete PZ (1999). Enterotoxigenic Escherichia coli (ETEC) in farm animals. Vet. Res. 30: 259-284.

Otte JM, Cario E and Podolsky DK (2004). Mechanisms of cross hyporesponsiveness to Toll-like receptor bacterial ligands in intestinal epithelial cells. Gastroenterology 126: 1054-1070.

Oyama J, Blais C Jr, Liu X, Pu M, et al. (2004). Reduced myocardial ischemia-reperfusion injury in toll-like receptor 4-deficient mice. Circulation 109: 784-789.

Penders J, Thijs C, Mommers M, Stobberingh EE, et al. (2010). Host-microbial interactions in childhood atopy: toll-like receptor 4 (TLR4), CD14, and fecal Escherichia coli. J. Allergy Clin. Immunol. 125: 231-236.

Qiu XT, Li YH, Li HJ, Yu Y, et al. (2007). Localization and tissue expression of swine Toll-like receptor 4 (TLR4). J. Agr. Biotechnol. 15: 37-40.

Roy MF, Lariviere L, Wilkinson R, Tam M, et al. (2006). Incremental expression of Tlr4 correlates with mouse resistance to Salmonella infection and fine regulation of relevant immune genes. Genes Immun. 7: 372-383.

Sun Q, Zheng Y, Liu Q and Cao X (2008). Rapamycin reverses TLR4 signaling-triggered tumor apoptosis resistance by disrupting Akt-mediated Bcl-xL upregulation. Int. Immunopharmacol. 8: 1854-1858.

Van Linthout S, Spillmann F, Graiani G, Miteva K, et al. (2011). Down-regulation of endothelial TLR4 signalling after apo A-I gene transfer contributes to improved survival in an experimental model of lipopolysaccharide-induced inflammation. J. Mol. Med. 89: 151-160.

Vogeli P, Meijerink E, Fries R, Neuenschwander S, et al. (1997). A molecular test for the detection of E. coli F18 receptors: a breakthrough in the struggle against edema disease and post-weaning diarrhea in swine. Schweiz. Arch. Tierheilkd. 139: 479-484.

Wang Q, Zhu H, Zhu J and Zhou LN (2011). Dynamic expression of Toll-like receptor 4 in septic acute lung injury in rats. J. Chin. Prac. Diagand. Ther. 25: 157-161.

Wieland CW, Florquin S, Maris NA, Hoebe K, et al. (2005). The MyD88-dependent, but not the MyD88-independent, pathway of TLR4 signaling is important in clearing nontypeable Haemophilus influenzae from the mouse lung. $J$. Immunol. 175: 6042-6049.

Wu SL, Yuan ZW, Ju HP, Huang XG, et al. (2007). Polymorphisms of the FUT1 gene M307 locus in post-weaning Sutai breed piglet and resistance to F18 fimbrial Escherichia coli in vitro. Chinese J. Prev. Vet. Med. 29: 783-787.

Yang H, Wei J, Zhang H, Song W, et al. (2010). Upregulation of Toll-like Receptor (TLR) expression and release of cytokines from mast cells by IL-12. Cell Physiol. Biochem. 26: 337-346.

Zhang X, Shan P, Qureshi S, Homer R, et al. (2005). Cutting edge: TLR4 deficiency confers susceptibility to lethal oxidant lung injury. J. Immunol. 175: 4834-4838. 